\title{
Processing data from new XFELs in cctbx.xfel and DIALS
}

Aaron S. Brewstera, Asmit Bhowmicka, Robert Bolotovskya, Derek Mendeza, and Nicholas K. Sautera

aLawrence Berkeley National Laboratory, Berkeley, CA 94720, USA (asbrewster@lbl.gov)

Over the last few years, new XFELs have come online, including the EuXFEL, PAL-XFEL, and SwissFEL, joining the LCLS and SACLA facilities in producing hard, coherent X-ray pulses used in serial crystallography. Each XFEL facility produces pulses differently, with different pulse-lengths, spectra, and repetition rates, recorded on new and complex detectors. We describe how we have adapted cctbx.xfel and DIALS to support these experiments using standards such as NeXus and providing user interfaces amenable to fast feedback of processing results. We also show new methods for determining the structure factors of small molecules from integrated reflections. 